\title{
MECHANICAL PROPERTIES OF A PHOTOPOLYMERIZABLE HYDROGEL FOR INTERVERTEBRAL DISC REPLACEMENT
}

\author{
A. Schmocker ${ }^{1}$, A. Khoushabi ${ }^{2}$, D. Pioletti ${ }^{3}$, P.E. Bourban², J.A. Månson ${ }^{2}$, C. Schizas ${ }^{4}$, C. Moser ${ }^{1}$ \\ ${ }^{1}$ Microengineering Department, Ecole Polytechnique Fédérale de Lausanne (EPFL), Switzerland \\ ${ }^{2}$ Institute of Materials, EPFL, Switzerland \\ ${ }^{3}$ Center of Translational Biomechanics, EPFL, Switzerland \\ ${ }^{4}$ Centre Hospitalier Universitaire Vaudois, Switzerland
}

andreas.schmocker@epfl.ch

\begin{abstract}
We report on the modelling and experimental validation of a photopolymerizable hydrogel for a Nucleus Pulposus replacement.
\end{abstract}

Keywords: Nucleus pulpous replacement, photopolymerized medical implant, injectable hydrogel, intervertebral disc testing

\section{Introduction}

Lower-back pain is experienced by 70 to $85 \%$ of the world's population once in their life. ${ }^{1}$ For intervertebraldisc-arthroplasty spinal-fusion remains the gold standard. ${ }^{2}$ Other currently available implants and treatments do not prove to be more efficient. ${ }^{3,4}$ Yet, more physiological solutions would strongly reduce the degree of invasiveness. Thus, an attractive option is to replacement the Nucleus Pulposus, the interior of the intervertebral disc. ${ }^{5}$ Photopolymerizable hydrogels are a viable option. During polymerization, the material's absorption and scattering coefficients change and directly influence local polymerization rates and hence the mechanical properties. By controlling the input light pattern, local material properties can be engineered, such as elastic moduli and swelling ratios to match the set of requirements for the replacement. Furthermore, the light necessary for the photopolymerization can be constrained to a small light guide to keep a surgical procedure as minimally invasive as possible.

\section{Methods}

Photopolymerizable hydrogels were injected or molded in their liquid form and then polymerized inside a tissue- or artificial cavity.
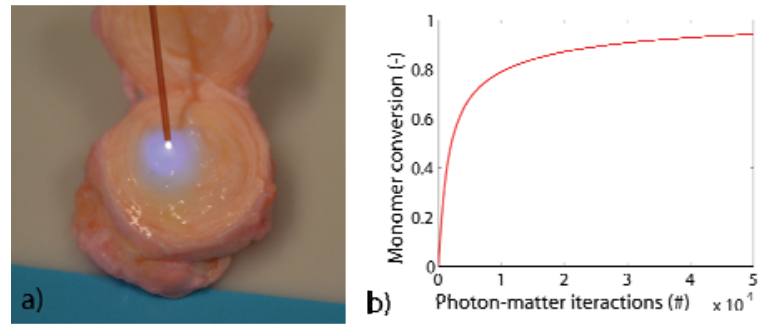

Figure 1: a) bovine intervertebral disc with gel sample illuminated by an optical light guide b) Simulation result: Monomer conversion during photopolymerization
Hence using an optical fiber gels of different volumes and shapes were polymerized (sample shown in Figure 1a). Quantitative scattering and absorption values as well as monomer conversion rates of the hydrogel sample were evaluated with a newly established Monte Carlo model (Figure 1b) for photopolymerization and then compared to photo-rheology measurements. Stress relaxation and fatigue behavior of polymerized samples were measured on a material testing machine to assess gels' short- and long term behaviors.

\section{Results}

3D polymerization patterns for different illumination configurations and associated material properties could be predicted. Swelling ratios and elastic moduli were measured as a function of monomer conversion. Preliminary results of hydrogel fatigue tests are presented in Figure 2 showing material-stiffening after repetitive cyclic testing, but no change in relaxation behavior.

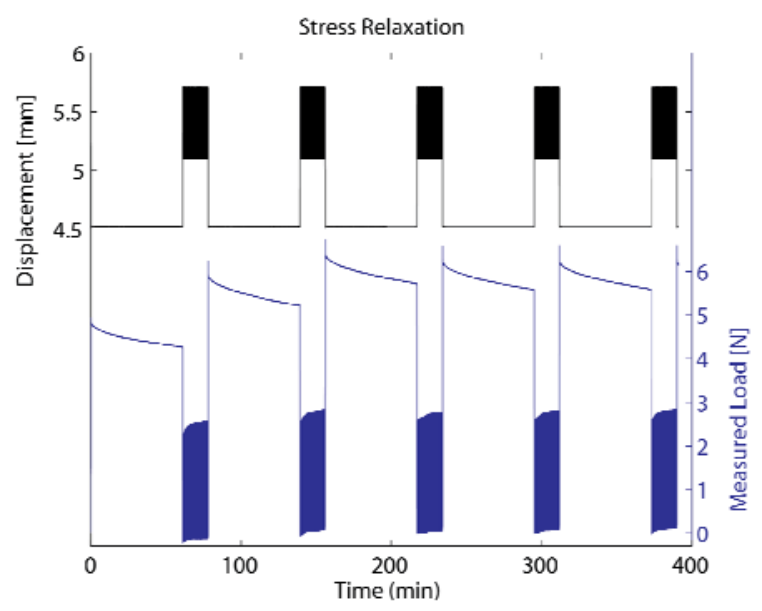

Figure 2: Five stress relaxations (duration: 1h) of a PEGDM sample-gel combined with cyclic testing (filled areas, duration: $17 \mathrm{~min}$, number of cycles: 1000)

\section{Discussion}

An approach is proposed, where properties like swelling ratios or elastic moduli can be tailored for in-situ implant design. However, to mimic the complexity of living tissues further testing and development are required. Photo-polymerized material strongly depend on the polymerization conditions and their environment ${ }^{6}$. Factors 
like local oxigen levels or light scattering within a tissue can heavily change polymerization-outcomes and thus final material properties.

\section{Acknowledgement}

Funding for this research was provided by the Swiss National Fund (\#10024003165465).

\section{Bibliography}

[1] Andersson, G.B., "Epidemiological features of chronic low-back pain.," Lancet 354(9178), 581-5 (1999).

[2] Zarghooni, K., Siewe, J., and Eysel, P., "State of the art of lumbar intervertebral disc replacement" Der Orthopädem 40(2), 141-7 (2011).

[3] Schizas, C., Kulik, G., and Kosmopoulos, V., "Disc degeneration: current surgical options" European cells \& materials 20, 306-15 (2010).

[4] Hu, R.W., Jaglal, S., Axcell, T., and Anderson, G., "A population-based study of reoperations after back surgery" Spine 22(19), 2265-2270; discussion 2271 (1997).

[5] Pelletier, M.H., Walsh, W.R., and South, N., Nucleus Replacement: Elsevier Ltd., 171-190 (2011).

[6] Anseth, K.S., Bowman, C.N., and Brannon-Peppas, L., "Mechanical properties of hydrogels and their experimental determination" Biomaterials 17(17), 164757 (1996). 